\title{
Saúde Mental: obstáculos e expectativas sentidos pelo médico de família
}

Ana Isabel Silva,* Ana Sofia Nogueira, ${ }^{* *}$ Rui Campos, ${ }^{* * *}$ Sandrina Martins****

\section{RESUMO}

Objectivos: Os obstáculos com que os médicos de família se deparam na sua prática clínica são uma fonte para mudanças e melhoria da qualidade na área da saúde mental a nível dos Cuidados Primários. Assim, pretendemos investigar os obstáculos e expectativas sentidos pelos médicos de família dos Agrupamentos de Centros de Saúde dos autores, na abordagem da patologia psiquiátrica.

Tipo de Estudo: Observacional, transversal e descritivo.

Local: Agrupamentos de Centros de Saúde dos investigadores.

População: Médicos de família

Métodos: Estudo realizado entre Julho e Novembro de 2011, através de um questionário de auto-preenchimento, elaborado pelos investigadores.

Resultados: Participaram 131 médicos neste estudo. Os principais obstáculos na abordagem da patologia psiquiátrica referiram-se ao tempo inadequado de consulta disponível (63\% consideram o tempo de consulta programada inadequado e $79 \%$ consideram o tempo de consulta não programada inadequado), à limitação no conhecimento de critérios de referenciação (49\%) e de técnicas de comunicação na consulta (40\%) e à relutância dos doentes na referenciação aos Cuidados Secundários (47\%). Destacaram-se, como principais expectativas, a criação de critérios de referenciação (97\%), melhoria do acesso a psiquiatras (60\%) e psicólogos (33\%), melhoria da interacção dos Cuidados Primários com o Serviço de Psiquiatria da área de referenciação (93\%), nomeadamente através de consultadoria (96\%) e sob a forma de reuniões com discussão de casos (73\%).

Conclusão: Este estudo demonstra a necessidade de se entender a patologia psiquiátrica como merecedora de maior tempo de consulta, de investir em formação pós-graduada e cursos na área da Saúde Mental e em promover sessões de esclarecimento e sensibilização para a patologia psiquiátrica, destinadas aos doentes.

Palavras-chave: Barreiras à Comunicação; Saúde Mental; Cuidados de Saúde Primários.

\section{INTRODUÇÃO}

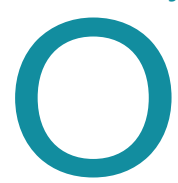

s distúrbios mentais são actualmente considerados um problema mundial e afectam todos os países, sociedades e grupos etários, com elevados custos sócio-económicos (cerca de $12 \%$ do total de anos de vida ajustados por incapacidade perdidos e $1 \%$ dos gastos totais em saúde). ${ }^{1}$ Diversos factores genéticos, biológicos, sociais e ambientais são hoje reconhecidos na sua origem, mas, até meados do século XX, os doentes com patologia psi-

* Interna de Medicina Geral e Familiar, USF Nova Salus.

**Interna de Medicina Geral e Familiar, USF São Félix da Marinha.

***Interna de Medicina Geral e Familiar, USF Nova Via.

****Especialista em MGF, UCSP Arouca. quiátrica sofriam em silêncio, sujeitos a discriminação, exclusão e estigma. ${ }^{1}$ Em 2001, a Organização Mundial de Saúde, sob o lema «Cuidar sim, excluir não», dedicou-se a avaliar o quadro da Saúde Mental no mundo e encontrou altas taxas de morbilidade e mortalidade associadas: 70 milhões de doentes sofrem de dependência do álcool, 24 milhões sofrem de esquizofrenia, 10 a 20 milhões tentaram suicídio e 1 milhão cometeo anualmente, sendo a principal causa de morte entre os jovens na maioria dos países desenvolvidos. Uma em cada quatro pessoas será afectada por um transtorno mental na sua vida. ${ }^{1}$ Prevê-se que em 2020 as doenças psiquiátricas sejam a segunda maior causa de gastos em saúde a nível mundial. ${ }^{1}$ 
Em Portugal, as doenças psiquiátricas constam dos motivos de consulta mais frequentes nos Cuidados de Saúde Primários (CSP), com uma prevalência de cerca de 50\%, encontrando-se metade destes doentes acompanhados exclusivamente pelo médico de família (MF). ${ }^{2}$

Existem inúmeros factores relacionados com o doente, o médico e a organização institucional que podem contribuir para um sub-diagnóstico e um sub-tratamento da patologia psiquiátrica. ${ }^{3,4,5,6} \mathrm{O} \mathrm{MF}$, ao cuidar do doente inserido numa família e numa comunidade, encontra-se numa posição privilegiada para identificar e tratar doentes com patologia psiquiátrica. No entanto, são muitas as dificuldades sentidas pelo MF na abordagem deste tipo de patologia. ${ }^{7,8}$ Tanto quanto os Investigadores sabem, a nível nacional não existe ainda nenhum estudo deste âmbito, que conste em alguma publicação indexada, razão pela qual consideram importante a sua realização, de modo a reorganizar, em colaboração com outros profissionais da área da saúde mental, ${ }^{9}$ estratégias de melhoria contínua da abordagem e seguimento destas patologias nos CSP.

\section{OBJECTIVOS}

O presente estudo tem como principais objectivos identificar obstáculos e expectativas sentidos pelos MF dos Agrupamentos de Centros de Saúde (ACeS) Grande Porto VII - Gaia e Grande Porto VIII - Espinho/Gaia.

\section{MÉTODOS}

Foi realizado um estudo observacional transversal e descritivo entre Julho e Novembro de 2011, nos ACeS Grande Porto VII - Gaia e Grande Porto VIII - Espinho/Gaia. O facto de ter sido utilizado um questionário não validado numa amostra de conveniência fez com que os autores optassem por realizar um estudo descritivo, pois um estudo analítico iria estar associado a diversos viéses. Futuramente, utilizando outro tipo de questionário e uma amostra aleatória, talvez seja possível elaborar um estudo analítico que obtenha resultados mais fidedignos e extensíveis a outras populações.

A população estudada foi o conjunto dos MF, com base numa amostra não aleatória de conveniência que compreende os MF dos ACeS acima enunciados.

Foram excluídos do estudo os médicos ausentes da
Unidade de Saúde Familiar (USF) ou da Unidade de Cuidados de Saúde Personalizados (UCSP) durante o período de recolha de dados, que decorreu entre 15 de Setembro e 15 de Outubro de 2011, e os questionários que não cumpriam as regras de preenchimento enunciados no questionário, nomeadamente a escolha de mais do que uma opção como resposta às diferentes questões, assim como a ausência de anonimato.

As variáveis dependentes estudadas consistiram nos obstáculos na abordagem da patologia psiquiátrica, avaliados através das questões 1-12 do questionário distribuído, nas expectativas quanto à necessidade de apoio profissionais de saúde, nomeadamente apoio de Psicologia, Nutrição ou Assistente Social na abordagem da patologia psiquiátrica (questão E2 e E2.1), assim como nas expectativas quanto a uma melhoria da interacção entre psiquiatras e MF e de que forma isso poderia ser realizado, via telefónica, postal, electrónica ou reuniões periódicas (questão E3 e E3.1) e nas expectativas quanto à elaboração de protocolos de referenciação aos Cuidados Secundários de Saúde (CSS) (questão E1 e E1.1). As variáveis independentes foram a idade, género, número de anos de especialidade, formação em saúde mental e existência de consulta de consultadoria de Psiquiatria regular na unidade de saúde. (Anexo 1)

Procedeu-se à recolha de informação, por meio de um questionário de auto-preenchimento, previamente submetido a teste piloto, desenvolvido pelos Investigadores e com o consentimento por escrito, livre e esclarecido dos participantes. O teste piloto foi realizado em duas Unidades Funcionais do ACeS Grande Porto VI - Porto Oriental, tendo sido respondido por 12 médicos. Essas respostas levaram à reformulação e simplificação de algumas questões. O estudo foi avaliado pelos ACeS Grande Porto VII - Gaia e Grande Porto VIII - Espinho/Gaia e Comissão de Ética da Administração Regional de Saúde (ARS) Norte.

O questionário foi entregue pessoalmente pelos Investigadores aos Coordenadores das respectivas USF/ /UCSP. Os dados foram recolhidos num envelope não identificado, destinado exclusivamente ao estudo em questão, e foram codificados e registados em base de dados informática - software Excel ${ }^{\circledR} 2010$. Obtiveram-se resultados descritivos da amostra (caracterização da amostra quanto ao género, idade, anos de especialida- 
de e formação pós-graduada) e das variáveis dependentes acima descritas.

O protocolo deste trabalho foi aprovado por unanimidade pela Comissão de Ética para a Saúde da ARS Norte, cujo Parecer n. ${ }^{\circ}$ 90/2011 foi emitido no dia 9 de Setembro de 2011. Previamente, o protocolo foi igualmente aprovado pelos ACeS envolvidos.

\section{RESULTADOS}

Dos 178 questionários distribuídos, incluíram-se no estudo 131, o que traduziu uma taxa de resposta de $73,6 \%$.

A maioria dos respondedores (65\%) era do género feminino e as idades situavam-se entre os 30 e os 64 anos, com predomínio do grupo etário dos 55 aos 59 anos $(\mathrm{n}=52)$.

Quanto ao número de anos de especialidade, verificou-se um pico entre os 25 e os 30 anos $(n=43)$. A formação pós-graduada em Saúde Mental foi referida por $53 \%$ dos MF.

Em relação aos obstáculos sentidos pelos MF na abordagem da Patologia Mental, o espaço físico e recursos disponíveis no local de trabalho foram considerados por $60 \%$ dos médicos inquiridos como $\mathrm{ra}$ ramente ou nunca constituem um obstáculo à sua prática clínica. No entanto, em relação ao tempo disponível na consulta programada para abordar a patologia psiquiátrica, $63 \%$ considerou que nunca ou raramente era adequado. No que respeita ao tempo de consulta não programada, $79 \%$ dos médicos considerou que o tempo nunca ou raramente era suficiente (Figura 1).

Constatou-se que o conhecimento sobre escalas de avaliação psiquiátrica ou guidelines era considerado ocasionalmente ou frequentemente útil por $72 \%$ e $67 \%$ dos MF respectivamente (Figura 2).

Nas questões relativas à terapêutica não farmacológica, constatou-se que $35 \%$ dos inquiridos considerava que o seu conhecimento nesta área nunca ou raramente limitava a prática clínica. No entanto, $41 \%$ referiu que nunca ou raramente a utilizava.

Relativamente à terapêutica farmacológica, $60 \%$ consideravam que o seu conhecimento farmacológico nunca ou raramente limitava a abordagem da patologia psiquiátrica e $73 \%$ utilizavam-na frequentemente (Figura 3).

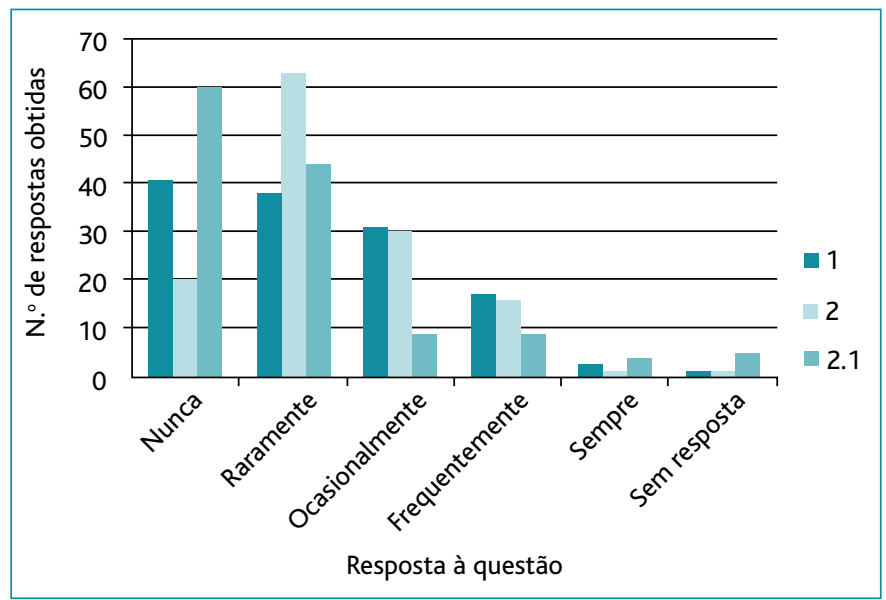

Figura 1. Respostas às questões 1,2 e 2.1:

1. O espaço físico e recursos disponíveis no seu local de trabalho são obstáculos à abordagem da patologia psiquiátrica?

2. Considera adequado o tempo de consulta programada para abordar a patologia psiquiátrica?

2.1. Considera adequado o tempo de consulta não programada para abordar a patologia psiquiátrica?

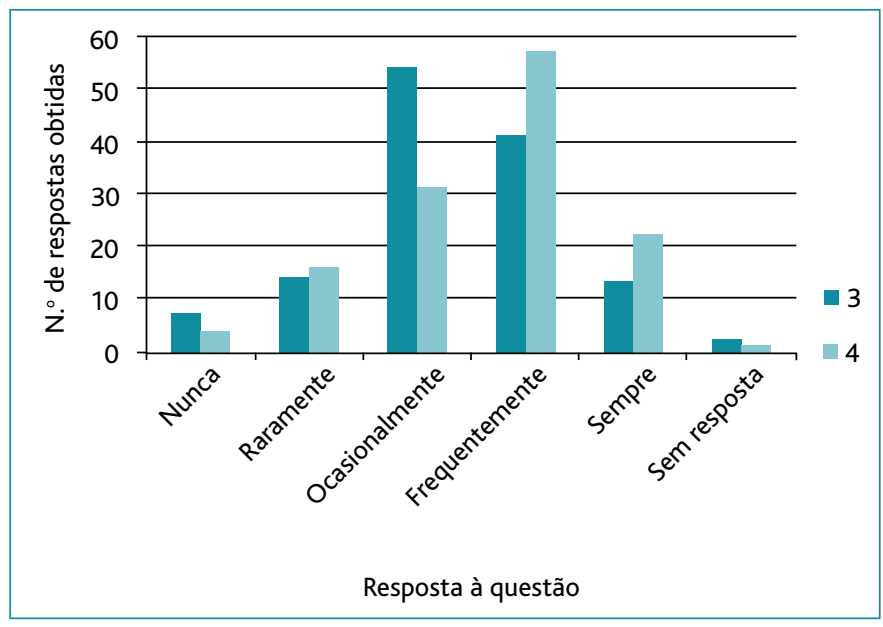

Figura 2. Respostas às questões 3 e 4 :

3. O seu conhecimento de escalas de avaliação de patologia psiquiátrica é útil na sua abordagem?

4. O seu conhecimento de guidelines sobre patologia psiquiátrica é útil na sua abordagem?

Os critérios de referenciação foram considerados por $50 \%$ dos médicos como uma ferramenta que facilitava frequentemente a abordagem desta patologia e $2 \%$ dos inquiridos considerava que os critérios de referenciação nunca eram úteis.

Dos médicos inquiridos, $49 \%$ não tinham conheci- 
mento da existência de critérios de referenciação para a consulta de Psiquiatria, sendo que 97\% destes médicos gostariam que esses critérios fossem criados.

Mais de $90 \%$ dos MF gostariam que houvesse uma melhoria na articulação com outros profissionais de saúde (psicólogo, nutricionista, psiquiatra ou assistente social), com maior capacidade de atendimento e menor tempo de espera até observação clínica, sendo que a melhoria na interacção com o psiquiatra foi referida por $60 \%$, com o psicólogo por $33 \%$, com o nutricionista por $5 \%$ e com o assistente social por $2 \%$ desses MF. A percentagem de MF que gostaria de uma melhoria na interacção com o Serviço de Psiquiatria foi de $93 \%$. Destes, $67 \%$ preferia as reuniões periódicas, $19 \%$ a via electrónica, $11 \%$ o contacto telefónico, $1 \%$ a via postal e $2 \%$ não responderam.

Dos médicos inquiridos, $80 \%$ referem não existir consultadoria na sua unidade funcional, pelo que $96 \%$ destes gostariam que a mesma existisse, sendo maioritariamente (73\%) sob a forma de reuniões para discussão de casos.

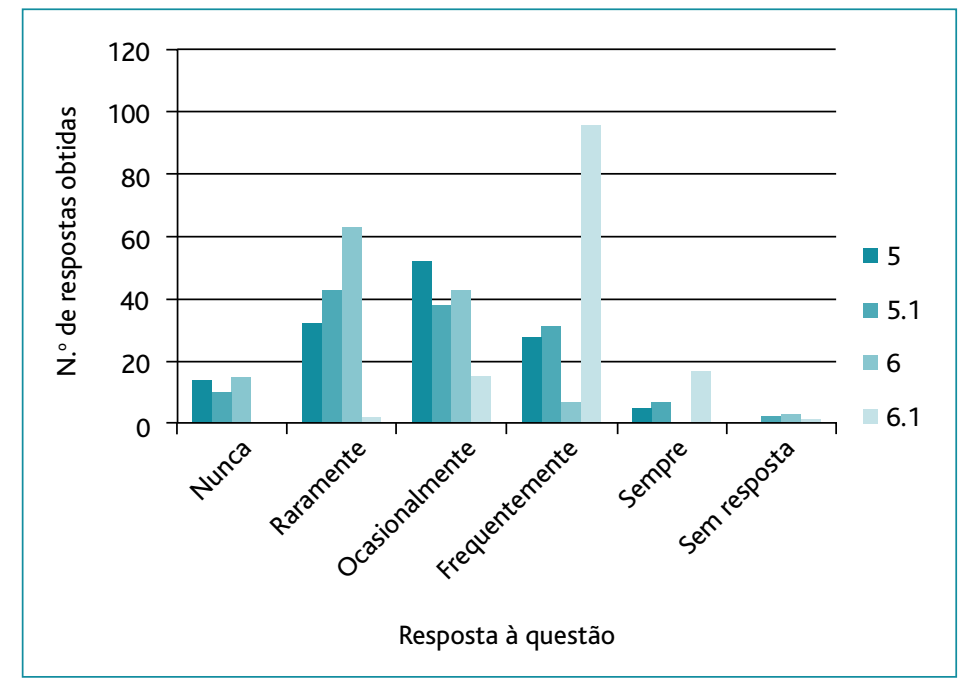

Figura 3. Respostas às questões 5, 5.1, 6 e 6.1:

5. O seu conhecimento sobre terapêuticas não farmacológicas específicas da patologia psiquiátrica limita na sua abordagem?

5.1. Utiliza essas terapêuticas não farmacológicas no tratamento de patologias psiquiátricas?

6. O seu conhecimento sobre terapêuticas farmacológicas específicas da patologia psiquiátrica limita na sua abordagem?

6.1. Utiliza essas terapêuticas farmacológicas no tratamento de patologias psiquiátricas?

\section{DISCUSSÃO}

A taxa de resposta do corrente estudo foi de $73,6 \%$, o que foi superior ao encontrado noutros estudos, nomeadamente no estudo de Lennox et al, ${ }^{5}$ que foi de $58 \%$.

Comparativamente ao estudo de Lennox et al, ${ }^{5} \mathrm{a}$ maioria dos respondedores era do género masculino (68\%), o que contrasta com os $35 \%$ do estudo actual. Nesse mesmo estudo a média de idades foi de 46,3 anos, enquanto no presente estudo, apesar de diferenças metodológicas, $60 \%$ apresentavam idade superior a 50 anos. O período de tempo em que os respondedores tinham prática clínica foi de 17,4 anos no estudo de Lennox et al, ${ }^{5}$ enquanto no estudo actual $54 \%$ tinham mais de 20 anos de prática clínica.

No que diz respeito à formação pós-graduada, 53\% dos respondedores referiram que a tinham, o que foi inferior aos $65 \%$ dos entrevistados do estudo de Lennox et al. ${ }^{5}$

O tempo de consulta foi uma das barreiras detectadas no presente estudo, o que foi de encontro ao obtido no estudo de Lennox et al, ${ }^{5}$ de Rijswijk et al ${ }^{6}$ e de Bar- ley et al. ${ }^{7}$ No estudo de Rijswijk et al, ${ }^{6}$ o tempo de consulta é considerado como uma importante barreira na realização de anamnese, diagnóstico de patologia mental e psico-educação. A necessidade de aumentar o tempo de consulta, nomeadamente através da marcação de dois períodos consecutivos de consulta ou através do aumento da frequência de consultas, foi uma das expectativas para melhorar a abordagem da patologia psiquiátrica descrita no estudo de Lennox et al. ${ }^{5}$

Em 93\% dos respondedores do estudo de Lennox et $a l,{ }^{5}$ a falta de formação/experiência foi considerada um obstáculo na abordagem da patologia psiquiátrica. Em relação a este ponto, Barley et al mostrou que os médicos percepcionaram a falta de conhecimento na área da saúde mental referindo a importância de mais formação. Smolders et $a l^{\beta}$ reportaram que a não adesão às guidelines por falta de tempo foi considerada um obstáculo na abordagem da patologia psiquiátrica. No presente estudo, $30 \%$ dos respondedores não consideram as guidelines de actuação na patologia psiquiátrica úteis na prática clínica. Segundo Addington et al, ${ }^{3}$ uma bar- 
reira importante para a implementação de guidelines em CSP é o facto de muitas destas serem originalmente desenvolvidas para aplicação em CSS.

No estudo de Rijswijk et al, ${ }^{6}$ os MF valorizam a importância do reconhecimento, diagnóstico e tratamento da ansiedade e depressão a nível dos CSP. No entanto, muitos manifestaram fortes reservas acerca da validade e utilidade dos conceitos do DSM-IV em CSP. Neste mesmo estudo a resistência em relação ao uso de antidepressivos, essencialmente a longo prazo, e o facto de que outros distúrbios psicossociais e co-morbilidades mentais podem ofuscar as características de depressão e ansiedade, eram vistos como barreiras para a aplicação das guidelines.

No presente estudo, apenas $35 \%$ dos inquiridos considerava que o seu conhecimento relativamente a terapêutica não farmacológica nunca ou raramente limitava a prática clínica. No entanto, $41 \%$ referiu que nunca ou raramente a utilizava, o que, segundo Rijswijk et al, ${ }^{6}$ pode ser explicado pela escassez de tempo na abordagem de patologia psiquiátrica.

O estudo de Barley et al referiu que os médicos, apesar de reconhecerem a eficácia dos fármacos, nem sempre seguiam as guidelines de prescrição e que essas prescrições apresentavam doses infraterapêuticas ou eram utilizadas por um curto período de tempo. Neste estudo, $60 \%$ dos entrevistadores não consideraram como barreira o seu conhecimento em relação à terapêutica farmacológica, sendo que $73 \%$ a utilizavam frequentemente, o que estava de acordo como o estudo de Barley et al, ${ }^{7}$ no qual o recurso à terapêutica farmacológica era a estratégia mais utilizada na abordagem da patologia psiquiátrica, na medida em que muitas vezes era a sua única opção. Isto porque ia de encontro às necessidades sentidas por mais de $90 \%$ dos respondedores do actual estudo, que gostariam que existisse uma melhoria da resposta de outros profissionais de saúde para abordarem a patologia psiquiátrica, nomeadamente, com o psiquiatra (60\%) e o psicólogo (33\%) e da interacção com o Serviço de Psiquiatria da área de referenciação (93\%). Também no estudo de Lennox et a ${ }^{5}$ foi referida a necessidade de uma melhoria da comunicação e interacção com outros profissionais de saúde. O actual estudo mostrou que a interacção com o Serviço de Psiquiatria deveria ser realizada preferencialmente através de reuniões periódicas.
No estudo de Lennox et al, ${ }^{5} 85 \%$ dos entrevistados consideraram que a comunicação na consulta era um dos obstáculos na abordagem da patologia psiquiátrica, o que diferiu do presente estudo em que apenas $40 \%$ considerou a comunicação na consulta como um obstáculo.

No estudo actual, $50 \%$ dos respondedores consideraram facilitador da abordagem da patologia psiquiátrica a existência de critérios de referenciação, o que vai de encontro com o estudo de Lennox et al, ${ }^{5} \mathrm{em}$ que o conhecimento inadequado dos entrevistadores em relação aos serviços/recursos disponíveis para referenciação dos doentes com esta patologia era considerado uma limitação. O estudo de Smolders et al referiu que a tendência para referenciar os doentes aos CSS era mais evidente nos respondedores mais velhos, o que poderá ir de encontro à tendência do presente estudo para os respondedores indicarem a referenciação como um elemento facilitador. Para além disso, o presente estudo mostrou que $49 \%$ dos respondedores não tinham conhecimento da existência de critérios de referenciação, tendo a maioria (97\%) indicado a necessidade da sua criação.

A baixa colaboração do doente em ser referenciado aos CSS foi considerada um obstáculo na abordagem da patologia psiquiátrica no estudo de Lennox et al, ${ }^{5} \mathrm{o}$ que foi referido por $47 \%$ dos respondedores do presente estudo. Quanto ao estigma, Barley et al concluíram que este era uma barreira na abordagem da patologia psiquiátrica, nomeadamente, em doentes mais velhos, tendência essa que não se observou no actual estudo.

O financiamento insuficiente foi considerado um obstáculo na abordagem da patologia psiquiátrica no estudo de Lennox et al, ${ }^{5}$ o que não se verificou no presente estudo.

Quanto aos pontos fortes deste estudo, destaca-se o facto de ter sido aplicado à totalidade da população em estudo com uma taxa de respostas elevada e ser o primeiro estudo realizado nos ACeS referidos e com questões orientadas para a interacção da articulação dos CSP com os CSS.

Quanto às limitações do presente estudo, salienta-se o facto de ter sido aplicado um questionário não validado (risco de viés de medição) com perguntas fechadas (risco de viés de informação). Apesar de ser um 
questionário anónimo, o facto de se terem incluído todos os profissionais médicos do ACeS pode causar algum viés de conveniência social. Por se tratar de um estudo retrospectivo, poderá também ter havido um viés de memória. A utilização de uma amostra de conveniência não permite que os resultados sejam extrapolados para outros ACeS...

A utilização da totalidade dos MF do ACeS para responder ao questionário deveu-se à pretensão que havia ao nível do Serviço de Psiquiatria em conhecer as necessidades dessa população com recurso a escrutínio geral das suas opiniões, pelo que foi utilizada essa metodologia em detrimento de outras técnicas qualitativas.

Deste modo, os principais obstáculos encontrados na abordagem da patologia psiquiátrica referiram-se ao tempo inadequado de consulta disponível, à limitação no conhecimento de critérios de referenciação e de técnicas de comunicação na consulta e a existência de alguma relutância dos doentes na referenciação aos CSS. Destacaram-se como principais expectativas para melhorar a abordagem da patologia psiquiátrica, a criação de critérios de referenciação, a melhoria do acesso a psiquiatras e psicólogos, a melhoria da interacção dos CSP com o Serviço de Psiquiatria da área de referenciação, nomeadamente, através de consultadoria, sob a forma de reuniões com discussão de casos.

Futuramente haverá a necessidade da realização do estudo numa amostra aleatória para possibilitar a extensão dos resultados ao nível nacional, a necessidade de investir em formação pós-graduada e em encontros/cursos na área da Saúde Mental e entender a patologia psiquiátrica como merecedora de um maior tempo de consulta/seguimento mais regular em detrimento da patologia física.

\section{REFERÊNCIAS BIBLIOGRÁFICAS:}

1. Relatório sobre a Saúde no Mundo 2001. Saúde Mental: nova concepção, nova esperança. Lisboa: Direcção-Geral da Saúde; 2001.

2. Relatório: Proposta de Plano de Acção para a Reestruturação e Desenvolvimento dos Serviços de Saúde em Portugal 2007-2016. Lisboa: Comissão Nacional para a Reestruturação dos Serviços de Saúde Mental em Portugal; 2006.

3. Addington D, Kyle T, Desai S, Wang J. Facilitators and barriers to implementing quality measurement in primary mental health care: Systematic review. Cam Fam Physician 2010 Dec; 56 (12): 1322-31.

4. ShieldT, Campbell S, Rogers A, Worrall A, Chew-Graham C, Gask L. Quality indicators for primary care mental health services. Qual Saf Health Care 2003 Apr; 12 (2): 100-6.

5. Lennox NG, Diggens JN, Ugoni AM. The General Practice care of people with intellectual disability: barriers and solutions. J Intellect Disabil Res 1997 Oct; 41 (Pt 5): 380-90.

6. Van Rijswijk E, van Hout $H$, van de Lisdonk E, Zitman F, van Weel C. Barriers in recognising, diagnosing and managing depressive and anxiety disorders as experienced by Family Physicians: a focus group study. BMC Fam Pract 2009 Jul 20; 10: 52.

7. Barley EA, Murray J, Walters P, Tylee A. Managing depression in primary care: A meta-synthesis of qualitative and quantitative research from the UK to identify barriers and facilitators. BMC Fam Pract 2011 Jun 9; 12: 47.

8. Smolders M, Laurant M, Verhaak P, Prins M, van Marwijk H, Penninx B, et al. Which physician and practice characteristics are associated with adherence to evidence-based guidelines for depressive and anxiety disorders? Med Care 2010 Mar; 48 (3): 240-8.

9. Mitchell G, Del Mar C, Francis D. Does primary medical practitioner involvement with a specialist team improve patient outcomes? A systematic review. Br J Gen Pract 2002 Nov; 52 (484): 934-9.

\section{CONFLITOS DE INTERESSE}

Os autores declaram não ter conflito de interesses.

\section{ENDEREÇO PARA CORRESPONDÊNCIA}

Ana Isabel Silva

Estrada da Granja, 114 Valbom, 4420-465 GONDOMAR

E-mail: anisabelfs@gmail.com

Recebido em 05-08-2013

Aceite para publicação em 15-10-2013 


\section{ABSTRACT}

\section{MENTAL HEALTH: OBSTACLES AND EXPECTATIONS OF FAMILY PHYSICIANS}

Aims: The approach to psychiatric disorders in Primary Health Care is a constant concern because its high prevalence, comorbidities, and associated mortality. Barriers to mental health care that Family Physicians find in their daily practice are a focus for quality improvement. The authors investigated the difficulties experienced by Family Physicians in mental health care and their expectations in managing psychiatric disorders.

Study Type: Observational

Location: Health Centre Groups in Northern Portugal

Population: Family Physicians

Methods: Study conducted between July and November 2011, using a questionnaire prepared by the investigators completed by the participants

Results: One hundred and thirty one physicians participated in the study. The main obstacles identified by family physicians in the management of psychiatric illness in primary care were inadequate time available for consultation (63\% of participants identified this issue for scheduled consultations and $79 \%$ for non-scheduled consultations), lack of knowledge of referral criteria (49\%), lack of communication skills (40\%), and the reluctance of patients for referral to hospital (47\%). The main expectations addressed were the creation of referral criteria (97\%), better access to psychiatrists (60\%) and psychologists (33\%), enhanced interaction between Primary Care and Psychiatric services available in the referral area (93\%) particularly through direct consultation (96\%) and through clinical case conferences (73\%).

Conclusion: This study demonstrates the need for additional consultation time for mental health in primary care, additional investment in training to acquire specific knowledge and skills for mental health care, and provision of case conferences to increase understanding and awareness in this field.

Keywords: Communication Barriers; Mental Health; Primary Health Care. 


\section{ANEXO I \\ QUESTIONÁRIO}

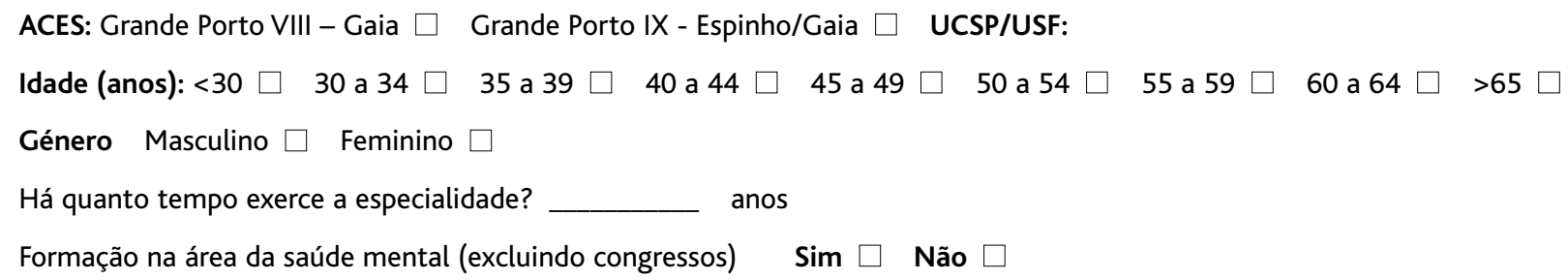

A patologia psiquiátrica pode colocar ao médico de família várias dificuldades. Classifique, de acordo com a sua experiência, qual a frequência com que se apresentam (assinalando com um $\mathbf{X}$ apenas uma opção por cada questão):

1. O espaço físico e recursos disponíveis no seu local de trabalho são obstáculos à abordagem da patologia psiquiátrica?

2. Considera adequado o tempo de consulta programada para abordar a patologia psiquiátrica?

2.1. Considera adequado o tempo de consulta não programada para abordar a patologia psiquiátrica?

3. O seu conhecimento de escalas de avaliação de patologia psiquiátrica é útil na sua abordagem?

4. O seu conhecimento de guidelines sobre patologia psiquiátrica é útil na sua abordagem?

5. O seu conhecimento sobre terapêuticas não farmacológicas específicas da patologia psiquiátrica limita na sua abordagem?

5.1. Utiliza essas terapêuticas não farmacológicas no tratamento de patologias psiquiátricas?

6. O seu conhecimento sobre terapêuticas farmacológicas específicas da patologia psiquiátrica limita na sua abordagem?

6.1. Utiliza essas terapêuticas farmacológicas no tratamento de patologias psiquiátricas?

7. Sente que o seu conhecimento de técnicas de comunicação na consulta limita a abordagem da patologia psiquiátrica?

8. Considera que critérios de referenciação facilitam a abordagem da patologia psiquiátrica?

9. Verifica relutância, por parte do doente com patologia psiquiátrica, em ser referenciado aos cuidados saúde secundários?

10. Os estigmas associados à saúde mental dificultam a sua abordagem na consulta?

11. Considera necessário haver maior motivação (por exemplo, incentivos) para a abordagem da patologia psiquiátrica?

12. Considera que os diferentes índices de burnout influenciam a abordagem que faz da patologia psiquiátrica? 
Expectativas:

Na tentativa de ultrapassar possíveis obstáculos e melhorar a abordagem das patologias psiquiátricas, pretende-se uma reflexão sobre os recursos disponíveis e as necessidades sentidas na sua área de trabalho (assinalando com um X apenas uma opção por cada questão)

E1 - Tem conhecimento da existência de critérios de referenciação da patologia psiquiátrica para os cuidados de saúde secundários?

$$
\text { Sim } \square \text { Não }
$$

E1.1 - Se respondeu não, gostaria que fossem criados critérios de referenciação da patologia psiquiátrica?

$$
\text { Sim } \square \text { Não }
$$

E2 - Gostaria que houvesse melhoria na resposta de outros grupos profissionais na sua área de referência?

$$
\text { Sim } \square \text { Não }
$$

E2.1 - Se respondeu sim, por parte de que profissional de saúde:

Nutricionista $\square$ Psiquiatra $\square$ Psicólogo $\square$ Assistente Social

E3 - Gostaria que houvesse melhoria na interacção entre os médicos de família e psiquiatras do hospital da área?

$$
\text { Sim } \square \text { Não }
$$

E3.1 - Se respondeu sim, como procederia para realizar essa interacção?

Contacto telefónico $\square \quad$ Via postal $\square \quad$ Via electrónica n Reuniões periódicas $\square$

E4 - Existe consultadoria de Psiquiatria na sua UCSP/USF?

$$
\text { Sim } \square \text { Não }
$$

E4.1 - Se não houver consultadoria prestada pelo serviço de Psiquiatria na sua UCSP/USF, consideraria importante a sua existência?

$$
\text { Sim } \square \text { Não }
$$

E4.2 - Se gostaria que houvesse consultadoria, de que forma a realizaria?

$$
\begin{gathered}
\text { Reuniões com discussão de casos } \square \text { Consulta partilhada } \square \text { Sessões teóricas } \\
\text { Outras } \square \text { (especifique): }
\end{gathered}
$$

Sugestões

O espaço que se segue destina-se a que possa deixar algumas sugestões que considere pertinentes e não tenham sido abordadas ao longo do questionário, de modo a melhorar a interacção entre o serviço de Psiquiatria da sua área de referência e os Cuidados de Saúde Primários. 